${ }^{1}$ Centro de Responsabilidad de Investigación, Instituto Nacional del Cáncer, Departamento Salud Pública y Epidemiología, Universidad de los Andes. Santiago, Chile. Escuela de Odontología, Facultad de Ciencias, Universidad Mayor, Chile.

${ }^{3}$ Centro de Responsabilidad Clínica de Rehabilitación, Instituto Nacional del Cáncer. Santiago, Chile.

${ }^{4}$ Equipo de Cabeza y Cuello, Instituto Nacional del Cáncer. Santiago, Chile.

${ }^{a}$ Médico Veterinario, Magíster en Salud Pública, Magíster en Bioestadística, Magíster en Administración y Gestión en Salud.

bAlumna de Odontología. 'Terapeuta ocupacional.

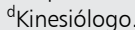
eFonoaudiólogo. ${ }^{f}$ Cirujano dentista.

Trabajo no recibió financiamiento. Los autores declaran no tener conflictos de interés

Recibido el 31 de agosto de 2017, aceptado el 12 abril de 2018.

Correspondencia a : Marcela Cárcamo I. Avenida Monseñor Álvaro del Portillo 12.455, Las Condes. Santiago, Chile. mcarcamo@uandes.cl

\section{Cáncer de cabeza y cuello: validación de cuestionario QLQ-H\&N35}

\author{
MARCELA CARCAMO ${ }^{1, a}$, VALENTINA CAMPO ${ }^{2, b}$, \\ DANIELA BEHRMANN ${ }^{2, b}$, CLAUDIA CELEDÓN $^{3, \mathrm{c}}$, ÁLVARO ALVEAR $^{3, \mathrm{~d}}$, \\ PABLO VÁSQUEZ ${ }^{3, \mathrm{e}}$, CRISTÓBAL ARAYA $^{2,4, \mathrm{f}}$

\section{Head and neck cancer: validation of the QLQ-H\&N35 quality of life questionnaire}

Background: Head and neck cancer affects esthetic and anatomical parameters, causing pain and functional impotence, affecting vital functions such as eating and breathing, hampering the quality of life of patients. EORTCQLQ-C30 questionnaire with its supplementary module QLQ-H\&N35 evaluates the quality of life of these patients. Aim: To statistically validate the QLQ-H \& N35 questionnaire in patients with head and neck cancer. Material and Methods: In a cross-sectional study, we studied patients with head and neck cancer who attended a rehabilitation unit. Reliability was measured using Cronbach's $\alpha$ and validity was determined by the diagnostic efficiency of the QLQ-H\&N35 scale as compared with the SF-36 quality of life survey. A Receiver Operational Characteristic (ROC) curve was generated. Results: The Cronbach's a global internal consistency of the questionnaire was $>0.70$ and its discrimination capacity was $74.2 \%$, which are considered acceptable. Conclusions: These results confirm the statistical validity of the QLQ-H\&N35 questionnaire, specifically for patients with head and neck cancer in Chile.

(Rev Med Chile 2018; 146: 578-584)

Key words: Head and Neck Neoplasms; Quality of Life; Validation Studies.
1 1 cáncer de cabeza y cuello (CCC) corresponde una neoplasia maligna que afecta principalmente al tracto aéreo digestivo superior y se subdivide según la región anatómica comprometida, clasificándose en cáncer de: cavidad oral, faringe (orofaringe, nasofaringe e hipofaringe), laringe (glótica, supraglótica e infraglótica), glándulas salivales, fosas nasales y senos paranasales. Corresponde al 3\% del cáncer en general, siendo el sexto más común en el mundo. Se ubica $40 \%$ en labio, cavidad oral y orofaringe, $25 \%$ en laringe, $15 \%$ en nasofaringe e hipofaringe y $20 \%$ en otros sitios anatómicos, como las glándulas salivales ${ }^{1}$.

La variedad histológica más frecuente es el carcinoma espinocelular (CEC). Dentro de su etiología, el uso de alcohol y de tabaco son los factores de riesgo más relevantes ${ }^{2}$, estando alre- dedor de $75 \%$ de los casos asociados a ellos ${ }^{3}$ y las personas que consumen ambos tienen un riesgo mayor debido a su efecto sinérgico ${ }^{4,5}$, llegando a tener un riesgo 10 a 20 veces mayor que aquellos pacientes que no fuman o no beben 6 . Se presenta preferentemente entre la 5 ta y 6 ta década y afecta más a hombres ${ }^{6-9}$. Otros factores asociados son los medio - ambientales, tales como los virus Epstein Barr, Virus papiloma humano, virus herpes simple, entre otros ${ }^{6}$.

En Chile, la incidencia para este cáncer, varía según la región de localización. Así, por ejemplo, para cáncer de labio y cavidad oral es de 1,6 por 100.000 habitantes en hombres y de 0,8 por 100.000 habitantes en mujeres y la mortalidad de 0,8 por 100.000 habitantes en hombres y de 0,3 por 100.000 habitantes en mujeres ${ }^{10}$. 


\section{Calidad de vida en salud}

En salud, la calidad de vida (CV) es una medida que relaciona la percepción del paciente frente a su salud o enfermedad, variando a lo largo del transcurso y resolución de ésta ${ }^{11,12}$.

En relación al cáncer en la región de CCC, se pueden ver afectados parámetros estéticos y anatómicos, pudiendo presentar dolor e impotencia funcional afectando funciones vitales, como comer y respirar. Todo esto repercute en aspectos psicológicos, como la autoestima, y la forma de interrelacionarse del paciente ${ }^{13}$.

Una forma de evaluar la CV en relación a la salud, es empleando cuestionarios. Para la adecuada utilización de estos, debe comprobarse la validez, para así ser aplicados a la población con la enfermedad en estudio ${ }^{14}$. Esto se garantiza, en primer lugar, por medio de la validación estadística del cuestionario, la que debe realizarse en cada país, adaptándose a las características poblacionales, $y$, en segundo lugar, a la validez lingüística que permite aplicar el instrumento al contexto social y cultural de este ${ }^{15}$.

Es así como la Organización Europea para Investigación y Tratamiento del Cáncer (EORTC) ha desarrollado diversos cuestionarios específicos de calidad de vida que se consideran completos y su formulación resulta culturalmente próxima a la idiosincrasia latina. La EORTC ha estructurado un cuestionario en base a 30 preguntas medidas mediante escala Likert, aplicable a todo paciente oncológico, ya adaptado culturalmente y validado en Chile ${ }^{16}$. Dentro de este cuestionario, existe como módulo suplementario el QLQ-H\&N35 para pacientes con CCC, que ha sido traducido a su versión en español, siendo validado por Arrarás et al (2001) en España. Esta versión ha sido modificada para su aplicación en la población chilena, sin embargo, no se ha validado estadísticamente en Chile.

Por lo tanto, el objetivo de este trabajo es validar estadísticamente la encuesta QLQ-H\&N35 en pacientes con CCC que se atienden en el Instituto Nacional del Cáncer (INC).

\section{Material y Método}

Diseño observacional analítico de corte transversal. La población de estudio está compuesta por pacientes con diagnóstico de CCC que se atienden en el INC. Este estudio incluye a pacientes, mayores de 18 años, con confirmación diagnóstica (clínica e histopatológica) de CCC, cáncer de piel y cáncer de tiroides con o sin tratamiento de su enfermedad. Estos dos últimos cánceres fueron considerados dada la severidad de los casos ingresados, implicando complejidad en los tratamientos.

Como criterios de exclusión, se consideraron a pacientes con presencia de tumores malignos secundarios concurrentes, con incapacidad para entender o completar los cuestionarios, y declaración de enfermedades críticas o desarrollo de complicaciones agudas en relación al tratamiento.

Se aplicó el cuestionario QLQ-C30 con el módulo suplementario QLQ-H\&N35 en conjunto con la encuesta SF-36, considerada esta última como Gold Standard (GS). Las encuestas fueron realizadas de manera presencial por estudiantes de 5to año de odontología de la Universidad Mayor en la Unidad de Rehabilitación del INC durante los meses de junio a diciembre del año 2016, las que fueron capacitadas previamente para la aplicación del instrumento. Los pacientes seleccionados consecutivamente, fueron informados sobre su participación asociada a la encuesta y se les solicitó su consentimiento informado para aplicar, analizar y publicar los datos.

El estudio fue aprobado por el Comité de Ética del Servicio de Salud Metropolitano Norte el día 3 de septiembre de 2015.

\section{Análisis estadístico}

\section{Cálculo de tamaño de muestra}

El tamaño muestral se calculó según la propuesta de Tabachnik y Fidell ${ }^{19}$, considerando que con el fin de obtener estimaciones fiables a través de análisis multivariado, el número de observaciones debe ser de 5 a 10 veces el número de variables del modelo. En este caso se estimó en 150 individuos, ya que QLQ-C30 incorpora 30 preguntas.

\section{Validación estadística}

Se realizó estadística descriptiva según el tipo y escala de la variable, reportando estadígrafos de tendencia central, dispersión y posición.

La confiabilidad de los cuestionario C30 y H\&N35, se evaluó mediante el coeficiente de $\alpha$ de Cronbach, y se estableció el nivel de confiabilidad según los criterios de George y Mallery ${ }^{17}$ 
(Tabla 1). Para evaluar la validez concurrente, se convirtieron los puntajes Linkert a valores entre 0 y 100 en cada uno de los instrumentos utilizados.

La validez se determinó por medio de la eficacia diagnóstica de la escala QLQ-H\&N35 en relación al GS (SF-36), que fue estimada mediante una curva Característica Operativa del Receptor (ROC) y los resultados fueron interpretados mediante los criterios de Hosmer y Lemeshow ${ }^{18}$.

Se consideraron puntos de corte enfocados en la especificidad para así pesquisar a los pacientes con mala calidad de vida.

Los datos recopilados fueron analizados con el software Stata V.13.1, considerando un nivel de significación de $5 \%$.

\section{Resultados}

Las encuestas fueron realizadas en 150 pacientes, 94 mujeres $(62,67 \%)$ y 56 hombres $(37,33 \%)$. La edad mediana fue de 61 años (20-88).

En relación al diagnóstico, se presentan en orden de frecuencia en la Tabla la y ordenados según su distribución por género en la Tabla $1 b$.

El 96,67\% de los pacientes había recibido o se encontraba en tratamiento como se puede apreciar en la Tabla 2. El mayor porcentaje de los pacientes $(88 \%)$ recibió tratamiento quirúrgico seguido por irradiación, quimioterapia y radioyodo y por último el 3,33\% de los pacientes se encontraba sin tratamiento al momento de realizar la encuesta Tabla 3.

\section{Validación de la escala}

\section{Análisis de confiabilidad}

En ambos cuestionarios se obtuvieron resultados de consistencia interna global aceptables $(\alpha$ de Cronbach fue sobre 0,70) ${ }^{17}$. La confiabilidad de la encuesta QLQ 30 por sí sola, fue de 93,35\%. El módulo suplementario H\&N35 tuvo una confiabilidad de 94,77\%, y ambos instrumentos de $96,21 \%$.

En el caso del cuestionario C30 los valores de $\alpha$ para las escalas multi-ítem se encontraron entre

Tabla 1b. Diagnósticos: distribución por género

\begin{tabular}{|lrr|}
\hline Diagnóstico & n & \multicolumn{1}{c|}{$\%$} \\
\hline Ca. Tiroides & 39 & \multicolumn{1}{c|}{26} \\
\hline CEC intraoral & 37 & 24,67 \\
\hline CBC & 16 & 10,67 \\
\hline Melanoma piel & 12 & 8,00 \\
\hline Tumor GS mayores & 9 & 6,00 \\
\hline Ca. Laríngeo / supraglótico / glótico & 8 & 5,33 \\
\hline CEC piel & 7 & 4,67 \\
\hline Ca. Orofaringe / rinofaringe / & 5 & 3,33 \\
hipofaringe & & \\
\hline CEC seno maxilar & 5 & 3,33 \\
\hline Osteosarcoma & 3 & 2,00 \\
\hline Linfoma no Hodgkin & 3 & 2,00 \\
\hline Tumor GS menor & 2 & 1,33 \\
\hline Otros sarcomas & 2 & 1,33 \\
\hline Ca. Paratiroides & 1 & 0,67 \\
\hline Melanoma mucoso & 1 & 0,67 \\
\hline Total & 150 & 100,00 \\
\hline
\end{tabular}

*Fuente de elaboración propia.

\begin{tabular}{|lccc|}
\hline Diagnóstico & Hombre & Mujer & Total \\
\hline Ca. Tiroides & 4 & 35 & 39 \\
CEC intraoral & 18 & 19 & 37 \\
\hline CBC & 7 & 9 & 16 \\
Melanoma piel & 4 & 8 & 12 \\
\hline Tumor GS mayores & 5 & 4 & 9 \\
\hline Ca. Laringeo / supraglótico / glótico & 5 & 3 & 8 \\
\hline CEC piel & 4 & 3 & 7 \\
\hline Ca. Orofaringeo / rinofaringe / & 3 & 2 & 5 \\
hipofaringe & & & \\
\hline CEC seno maxilar & 2 & 3 & 5 \\
\hline Osteosarcoma & 1 & 2 & 3 \\
\hline Linfoma no Hodgkin & 1 & 2 & 3 \\
\hline Tumor GS menor & 0 & 2 & 2 \\
\hline Otros sarcomas & 0 & 2 & 2 \\
\hline Ca. Paratiroides & 1 & 0 & 1 \\
\hline Melanoma mucoso & 1 & 0 & 1 \\
\hline Total & 56 & 94 & 150 \\
\hline *Fuente de elaboración propia. & & & \\
\hline
\end{tabular}

*Fuente de elaboración propia. 
Tabla 2. Aplicación de la encuesta en relación al período de tratamiento

\begin{tabular}{|lcc|}
\hline Período & Frecuencia & \% \\
\hline Previo & 5 & 3,33 \\
\hline Durante & 3 & 2,00 \\
\hline Posterior & 142 & 94,67 \\
Total & 150 & 100,00 \\
\hline
\end{tabular}

Tabla 3. Tratamientos oncológicos de muestra en estudio

\begin{tabular}{|lcc|}
\hline Tratamiento & Frecuencia & \% \\
\hline Operado & 132 & 88,00 \\
\hline Irradiado & 45 & 30,00 \\
\hline Quimioterapia & 16 & 10,67 \\
Radioyodo & 14 & 9,33 \\
\hline Sin tratamiento & 5 & 3,33 \\
\hline
\end{tabular}

*Existen pacientes que recibieron terapias combinadas como esquema terapéutico.
0,56 para la escala de función cognitiva, y 0,87 para las escalas de salud global y funcionamiento de roles. En el caso de la encuesta H\&N35 la mayor confiabilidad se obtuvo en la escala de "problemas con comidas sociales" con un valor de 0,78 y la menor en la escala de "problemas sensitivos" con un valor de 0,13 (Tabla 4).

\section{Análisis de validez}

La capacidad de discriminación fue de 74,2\%, lo que se considera aceptable. Se estableció como puntos de corte valores entre 40 y 45 , obteniendo especificidades que fluctúan entre $85,88 \%$ y 90,59\% (Figura 1).

\section{Discusión}

En Chile, la CV es considerada y evaluada cada vez con más frecuencia en las diversas áreas de la salud, sin embargo, muchas veces su evaluación es a través de cuestionarios no específicos o a través de encuestas que no han sido validadas ${ }^{16}$.

En el presente estudio, los datos epidemiológi-

Tabla 4. Consistencia interna de las escalas multi-ítem C30 y H\&N35

\begin{tabular}{|c|c|c|c|c|c|}
\hline & Escala de síntomas & ítems & Media & Desv. Est & Alpha de cronbach \\
\hline \multirow[t]{7}{*}{ H\&N35 } & Dolor & 4 & 22.83 & 25,71 & 0,75 \\
\hline & Tragar & 4 & 22.83 & 25,97 & 0,71 \\
\hline & Problemas sensitivos & 2 & 21.55 & 30,74 & 0,13 \\
\hline & Problemas para hablar & 3 & 27.11 & 27,80 & 0,77 \\
\hline & Problemas con comidas sociales & 4 & 25.28 & 29,35 & 0,78 \\
\hline & Problemas con contactos sociales & 5 & 19.42 & 23,20 & 0,77 \\
\hline & Menos sexualidad & 2 & 35.44 & 38,19 & 0,29 \\
\hline \multirow[t]{11}{*}{ C30 } & Salud Global & 2 & 69 & 23,11 & 0,87 \\
\hline & Escalas funcionales & & & & \\
\hline & Funcionamiento físico & 5 & 83.38 & 19,07 & 0,80 \\
\hline & Funcionamiento de roles & 2 & 76.44 & 28,97 & 0,87 \\
\hline & Funcionamiento emocional & 4 & 70.44 & 24,09 & 0,83 \\
\hline & Función cognitiva & 2 & 79.55 & 24,28 & 0,56 \\
\hline & Función social & 2 & 75.89 & 27,95 & 0,83 \\
\hline & Escala de síntomas & & & & \\
\hline & Fatiga & 3 & 30.15 & 23.36 & 0.76 \\
\hline & Náusea y vómito & 2 & 8 & 19.08 & 0.76 \\
\hline & Dolor & 2 & 31 & 28.77 & 0.81 \\
\hline
\end{tabular}




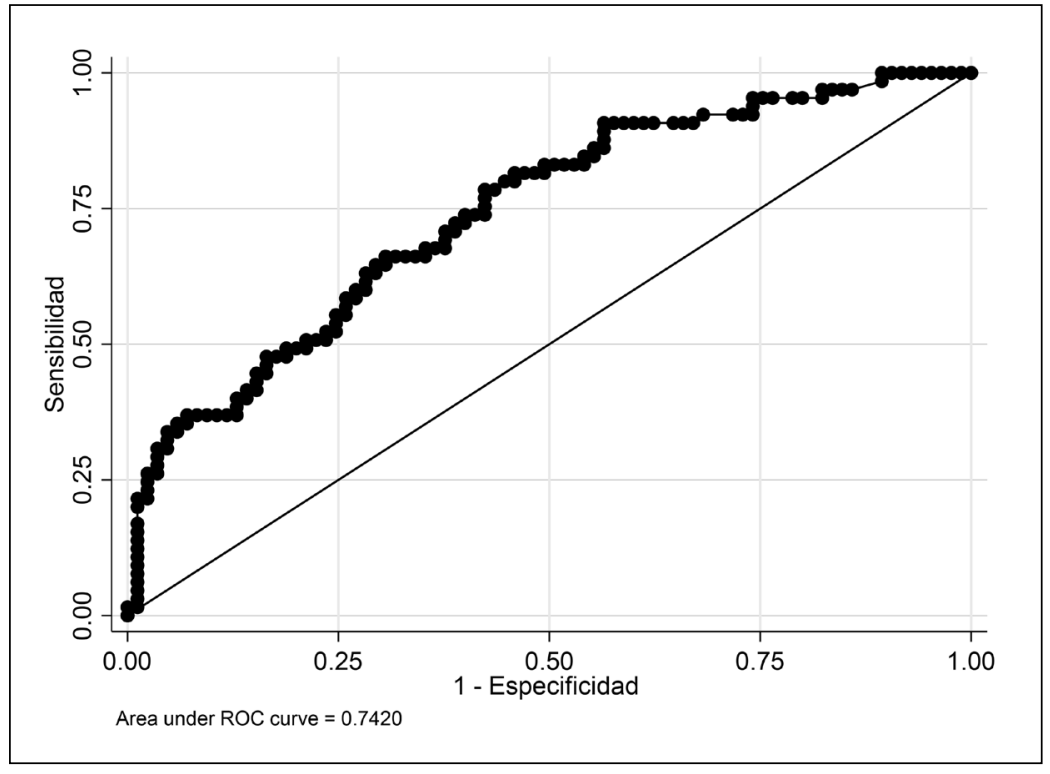

Figura 1. Análisis ROC EORTC QLQH\&N35/SF-36v2. cos presentaron resultados similares a lo descrito en la literatura, destacando que el cáncer de mayor prevalencia de la región de CCC es el de tiroides con mayor asociación al sexo femenino9. En el caso de CCC, el CEC correspondió a la variedad histológica más frecuente ${ }^{3}$, y hubo una brecha muy estrecha en su frecuencia de presentación entre los dos géneros, lo que se condice con la literatura, en la cual se relata que este cáncer ya no tiene una predicción marcada por el género masculino y que ha habido un aumento considerable en la incidencia del CEC en mujeres en los últimos años, lo que se asocia al aumento de mujeres fumadoras ${ }^{6-9}$.

En el presente estudio, los resultados obtenidos en la validación de QLQ-H\&N35 son comparables a los que se demostró en otros países, como México $^{20}$, España ${ }^{21}$, Polonia $^{22}$ y Japón ${ }^{23}$. En todos estos países el módulo H\&N35 tuvo resultados de consistencia interna aceptables $(\alpha>0,70)$, tanto al analizarse por sí sola como en conjunto al cuestionario C30.

En el análisis de confiabilidad de los ítems múltiples, la escala de "función cognitiva" de la encuesta C30 tuvo un valor menor al aceptable $(0,56)$, obteniendo valores por sobre 0,7 en el resto de las escalas. Al igual como se demostró en la validación de $\mathrm{México}^{20}$, donde obtuvieron un valor de 0,22 en esta escala, y valores sobre aceptables en todas las demás.
En el caso de la encuesta H\&N35, dos escalas obtuvieron valores bajo lo aceptable, la escala de "problemas sensitivos" y la de "menos sexualidad".

La menor confiabilidad se obtuvo en la escala de "problemas sensitivos" con un $\alpha$ de Cronbach de 0,13 . Esto es comparable a lo observado en México $^{20}$, donde esta escala tampoco obtuvo una confiabilidad aceptable y fue también la de menor valor $(0,49)$.

Por otro lado, en Japón y Polonia la confiabilidad de esta misma fue aceptable. Aunque en el caso de Polonia, fue también la de menor valor $(0,72)$, pero todas las escalas obtuvieron coeficientes $\alpha$ de Cronbach mayores a 0,70.

La segunda escala que no obtuvo una confiabilidad aceptable en este estudio fue la de "menos sexualidad". En contraste, a lo ocurrido en Japón ${ }^{23}$, donde esta escala obtuvo el mayor coeficiente $\alpha$ de Cronbach $(0,93)$.

Los resultados del estudio muestran una mayor similitud con lo que se describe por Carrillo et al. $(2013)^{20}$ en la validación de México, lo que puede guardar relación con que hay una mayor cercanía geográfica y cultural con esta población.

El análisis de confiabilidad utilizado es aplicable solo a escalas de ítems múltiples (más de una pregunta), y el valor $\alpha$ de Cronbach es sensible al número de preguntas de la escala. Todos los va- 
lores bajos se presentaron en escalas compuestas por 2 preguntas, lo que no reflejan necesariamente que estas escalas no midan de manera aceptable.

La encuesta $\mathrm{H} \& \mathrm{~N} 35$ por si sola tiene una confiabilidad que es alta en la predicción de la $\mathrm{CV}$ $(94,7 \%)$, lo que sugiere que, al implementarse sola, sin la encuesta C30, cumplirá el rol de evaluar la CV. A pesar de eso, se recomienda su aplicación en conjunto ${ }^{16}$, ya que ambos cuestionarios se complementan abarcando más áreas y recopilando información de utilidad para el tratamiento como también en el seguimiento del paciente. Esto se ve reflejado en los resultados obtenidos en nuestro estudio, donde se obtuvo mayor confiabilidad al aplicar las dos encuestas juntas (96,21\%).

En relación a la capacidad de discriminación de la escala H\&N35, determinada por el análisis de la curva ROC, fue de $74,2 \%$, lo que se considera aceptable. Esto la convierte en un instrumento útil para pesquisar la buena/mala CV de los pacientes. No hay muchos artículos de la validación de la encuesta H\&N35 que determinen la capacidad de discriminación mediante la curva ROC. Sin embargo, en un estudio realizado en Chile para validar el módulo específico de cáncer de mama desarrollado por la EORTC, el BR-23, realizaron el análisis de ROC utilizando el mismo Gold Standard (SF-36) para contrastar la encuesta. La capacidad de discriminación obtenida en este estudio fue de $0,93^{16}$.

La diferencia en la capacidad de discriminación entre ambas encuestas a favor del cuestionario BR23, puede deberse a que a nivel de CCC se presenta una mayor diversidad de neoplasias malignas, lo que hace que sean muy variados los efectos de éstas sobre la $\mathrm{CV}$, siendo difícil abarcarlos todos y estandarizarlos en una sola encuesta.

Además, establecieron puntos de corte según la especificidad, al igual que en el presente estudio. Se definió así para lograr descartar adecuadamente a aquellos pacientes con buena CV, y favorecer la pesquisa de aquellos con mala $\mathrm{CV}$, ya que estos últimos son el principal objetivo de la aplicación de la encuesta para poder ser intervenidos mejorando el tratamiento y reforzando el apoyo en estos paciente ${ }^{24}$.

Entre las limitaciones encontradas al realizar el estudio, está la gran cantidad de preguntas y el tiempo que demandó contestar las tres encuestas, lo que ocasionó que algunos pacientes se saltaran preguntas, invalidando estos cuestionarios para el estudio. Así también, la población es seleccionada por conveniencia, dada las condiciones del establecimiento (población asignada).

Por otro lado, no fue posible realizar la encuesta en pacientes hospitalizados en Unidad de Cuidados Intensivos o estados más graves, ya que requiere de mayor tiempo y colaboración de otros profesionales. Esto se condice con la literatura, en la que se describe que los pacientes más graves son menos asequibles a las evaluaciones, lo cual constituye un sesgo en los resultados ${ }^{25}$.

Por último, la utilización de este tipo de herramientas podría ser de ayuda tanto para el equipo médico como para el paciente y quienes cuidan de éste último permitiendo pesquisar aquellos pacientes con mala calidad de vida y permitiendo enfocar de mejor manera sus tratamientos y rehabilitación.

Agradecimientos: A todos los funcionarios de la Unidad de Rehabilitación y al Equipo de Cabeza y Cuello, del Instituto Nacional del Cáncer, Chile.

\section{Referencias}

1. Abreu de Aquino RC, de Lima LT, Luiza M, de Menezes CX, Regina C, Rodrigues M. Epidemiologic Aspects Of Mortality From Oral Cancer: Understanding The Risks To Enable The Early Detection Of Changes In Communication. Rev Cefac 2015; 17 (4).

2. Neville BW, Day TA. Oral cancer and precancerous lesions. CA Cancer J Clin 2002; 52 (4): 195-215.

3. Escribano-Bermejo M, Bascones-Martínez A. Leucoplasia oral: Conceptos actuales. Av En Odontoestomatol 2009; 25 (2): 83-97.

4. Antón M-S, Somacarrera Pérez ML. Cáncer oral: genética, prevención, diagnóstico y tratamiento. Revisión de la literatura. Av En Odontoestomatol 2015; 31 (4): 247-59.

5. Pelucchi C, Gallus S, Garavello W, Bosetti C, La Vecchia C. Cancer risk associated with alcohol and tobacco use: focus on upper aero-digestive tract and liver. Alcohol Res Health 2006; 29 (3): 193-9.

6. Shah JP, Patel SG, Singh B. Jatin Shah's head and neck surgery and oncology: [get full access and more at ExpertConsult.com]. 4. ed. Philadelphia. Pa: Elsevier, Mosby; 2012. 838 p.

7. Morbini P, Benazzo M. Human papillomavirus and head and neck carcinomas: focus on evidence in the babel of published data. Acta Otorhinolaryngol Ital 2016; 36 (4): 249-58. 
8. Stewart BW, Wild CP. World Cancer Report 2014. Lyon: International Agency for Research on Cancer/World Health Organization; 2014.

9. Cardemil F. Epidemiología del carcinoma escamoso de cabeza y cuello. Rev Chil Cir 2014; 66 (6): 614-620.

10. Globocan 2012. International Agency for Research on Cancer (IARC), World Health Organization (WHO).

11. Nazar G, Platzer L, González I, et al. Evaluación De Calidad De Vida En Pacientes Tratados Por Cáncer De Laringe. Rev. Otorrinolaringol. Cir Cabezacuello, 2004;64:190-198.

12. Urzúa A. Calidad de vida relacionada con la salud: elementos conceptuales. Rev Med Chile 2010; 138: 358-65.

13. Almeida AF, Alves RC, Felix JD, Castro DS, Zandonade E, Rocha RM. Qualidade de vida das pessoas acometidas por câncer no trato aerodigestivo superior em um Hospital Universitário. Rev Bras Cancerol 2013; 59(2): 229-37.

14. Ferriols Lisart R, Ferriols Lisart F, Alós Alminana M, Magraner Gil J. Calidad de vida en oncología clínica. Farm Hosp 1995; 19 (6): 315-22.

15. de Yébenes Prous MJG, Salvanés FR, Ortells LC. Validación de cuestionarios. Reumatol Clin 2009; 5 (4): 171-7.

16. Irarrázaval ME, Rodríguez PF, Fasce G, Silva FW, Waintrub $\mathrm{H}$, Torres $\mathrm{C}$, et al. Calidad de vida en cáncer de mama: validación del cuestionario BR23 en Chile. Rev Med Chile 2013; 141 (6): 723-34.

17. George D, Mallery, P. SPSS/PC+ step by step: A simple guide and reference. Belmont, CA. Estados Unidos Wadsworth Publishing Company 1995.
18. Hosmer D, Lemeshow S. Applied Logistic Regression. New York: Wiley. 2013.

19. Tabachnick BG, Fidell LS. Using multivariate statistics. Boston: Allyn \& Brown, 2001.

20. Carrillo JF, Ortiz-Toledo MA, Salido-Noriega Z, Romero-Ventura NB, Ochoa-Carrillo FJ, Oñate-Ocaña LF. Validation of the Mexican Spanish version of the EORTC QLQ-H\&N35 instrument to measure health-related quality of life in patients with head and neck cancers. Ann Surg Oncol 2013; 20 (5): 1417-26.

21. Arraras JI, Arias F, Tejedor M, Vera R, Pruiá E, Marcos $\mathrm{M}$, et al. El cuestionario de Calidad de Vida para tumores de cabeza y cuello de la EORTC QLQ-HFN35. Estudio de validación para nuestro país. Oncologia 2001; 24 (10): 482-91.

22. Tomaszewska IM, Glowacki R, Paradowska D, Walocha E. Preliminary psychometric validation of the Polish version of the EORTC head and neck module (QLQH\&N35). Folia Med Cracov 2013; 53 (1): 55-63.

23. Toth G, Sakaguchi T, Mikami Y, Hirose H, Tsukuda M. A pilot study of the translation, cultural adaptation and validation of the EORTC head and neck cancer quality of life questionnaire module (QLQ-H\&N35) for use in Japan. Auris Nasus Larynx 2005; 32 (2): 175-83.

24. Montazeri A. Quality of life data as prognostic indicators of survival in cancer patients: an overview of the literature from 1982 to 2008. Health Qual Life Outcomes 2009; 7: 102.

25. Grau J. Calidad de vida y salud: problemas actuales en su investigación. Boletín Latinoamericano de psicología de la salud 2009. 